\title{
Decay Reactions of Free Radicals in Irradiated Polyethylene and Diffusion-Controlled Processes
}

\author{
Shigetaka Shimada and Hisatsugu Kashiwabara \\ Materials Research Laboratory, Nagoya Institute of Technology, \\ Gokiso-cho, Showa-ku, Nagoya, Japan. \\ (Received December 10, 1973) \\ KEY WORDS Decay Reaction / Free Radical / Alkyl Radicals / \\ Polyethylene / Diffusion-Controlled Process /
}

The decay of free radicals in irradiated polymers have been investigated by many authors, and the decay reactions have been said to be second order ${ }^{1}$ in some case and first order ${ }^{2}$ in other cases. However, further improvement seems to be necessary for each of the studies. In this paper, it will be shown that the analysis of the decay reaction based on diffusion controlled reaction theory can make much more reasonable interpretation of the data obtained in the case of polyethylene. Also, we will show that the radical concentration in the region, in which the decay reaction proceeds, must be considered instead of the overall concentration in the matrix polymer.

In the analysis of the data we applied the diffusion-controlled reaction theory of Waite. ${ }^{3}$ According to his theory, concentration of the reactant at time $t, C(t)$, can be expressed by eq 1 for the case of uniform initaial distribution of the reactants

$$
\frac{1}{C(t)}=\frac{1}{C_{0}}+K\left[1+\frac{\sqrt{2} r_{0}}{(\pi D t)^{1 / 2}}\right] t
$$

where $C_{0}$ means the initial value of $C(t), D$ stands for the diffusion constant, $r_{0}$ for the capture radius, and $K$ equals $8 \pi r_{0} D$. The capture radius is defined as the distance between the reactants at which recombination occurs immediately. This equation is valid when the reaction is bimolecular, $A+B \rightarrow A B$, and the initial concentrations of $A$ and $B$ are equal.

Usually, a polymer matrix which traps the free radicals can be considered to be a multiphase system, say, amorphous and crystalline.
Therefore, the concentration of the free radicals must be represented as

and

$$
\begin{aligned}
& C=C^{\prime}+C^{\prime \prime} \\
& C_{0}=C_{0}{ }^{\prime}+C_{0}{ }^{\prime \prime}
\end{aligned}
$$

for the case of two-phase system, where $C^{\prime}$ and $C^{\prime \prime}$ are the concentrations of the radicals in the crystalline and amorphous regions, respectively. In the analysis of this paper, we assumed the followings: Though the concentrations of the radicals are not uniform throughout the whole system as indicated in eq 2 , decay reaction in each of two phases can occur with uniform initial concentration in each of the respective phases. This means that the free radicals in the respective phases decay with a diffusion constant which is characteristic for each of the respective phases and no diffusion of radicals occurs between the different phases. This assumption seems reasonable for the actual polymer system. Equation 1 can be rewritten in the following forms with some constants $A, A^{\prime}$ and $B$.

$$
\begin{aligned}
\frac{C_{0}}{C} & =1+B\left(1+\frac{A^{\prime}}{\sqrt{t}}\right) \\
& =1+A \sqrt{t}+B t
\end{aligned}
$$

And, by use of the expressions in eq 2, we have the following equations instead of eq 3

$$
\frac{C_{0}-C_{0}{ }^{\prime \prime}}{C-C_{0}{ }^{\prime \prime}}=\frac{1-a}{x-a}=1+B\left(1+\frac{A^{\prime}}{\sqrt{t}}\right) t
$$

or

$$
x=\frac{1}{B\left(1+\left(A^{\prime} / \sqrt{t}\right)\right)} \frac{1-x}{t}+a
$$


where

$$
x=\frac{C}{C_{0}}, \quad \text { and } \quad a=\frac{C_{0}^{\prime \prime}}{C_{0}} .
$$

These equations lead to the following: (I) Equation 3 shows a reaction behaving like a second order for longer $t$ at which time the quantity $A^{\prime} / \sqrt{t}$ can be negligible compared to unity. We can see that most of the plots of inverse concentration against time show a deviation from a linear plot for a shorter duration of time as shown in many papers concerning the free-radical decay in polymers. (II) Equation 3 indicates to us that a plot of inverse concentration $v s$. time at large $t$ can give us the value of $B$ and plot of inverse concentration vs. $\sqrt{t}$ at very small $t$ can give us the value of $A$ approximately for the case of a one component system. A similar estimation for the much complicated two-phase system can be made by the application of eq 4 and 5 . (III) When the free radicals are trapped in one phase only, quantity $a$ in eq 5 must vanish.
Based on this information, the following experiment was carried out: A sample of solutiongrown polyethylene was prepared from $0.1-\%$ xylene solution of Sholex 6050. Crystallization was made at $85^{\circ} \mathrm{C}$ for a week. The sample was $\gamma$-irradiated by Co-60 up to the dose of $\mathbf{1 . 2}$ Mrad in vacuo at liquid nitrogen temperature. The irradiated sample was subjected to a heat treatment at $27^{\circ} \mathrm{C}$ for a considerable duration of time. The ESR spectrum observed appeared to be the spectrum from alkyl-type free radicals trapped in the crystalline region. This was confirmed by the disappearance of the broad component admixed in the spectrum observed immediately after the irradiation. The plots based on eq 4 and 5 for this case also indicate that we can apply eq 3 as mentioned later. Free radical decays at $27^{\circ} \mathrm{C}$ and several other temperature was observed and the intensities of the ESR spectra were plotted against the time of storage at the respective temperatures.

If we make the plot of $x v s .(1-x) / t$, extrapolation of the curve to $(1-x) / t=0$ gives us the

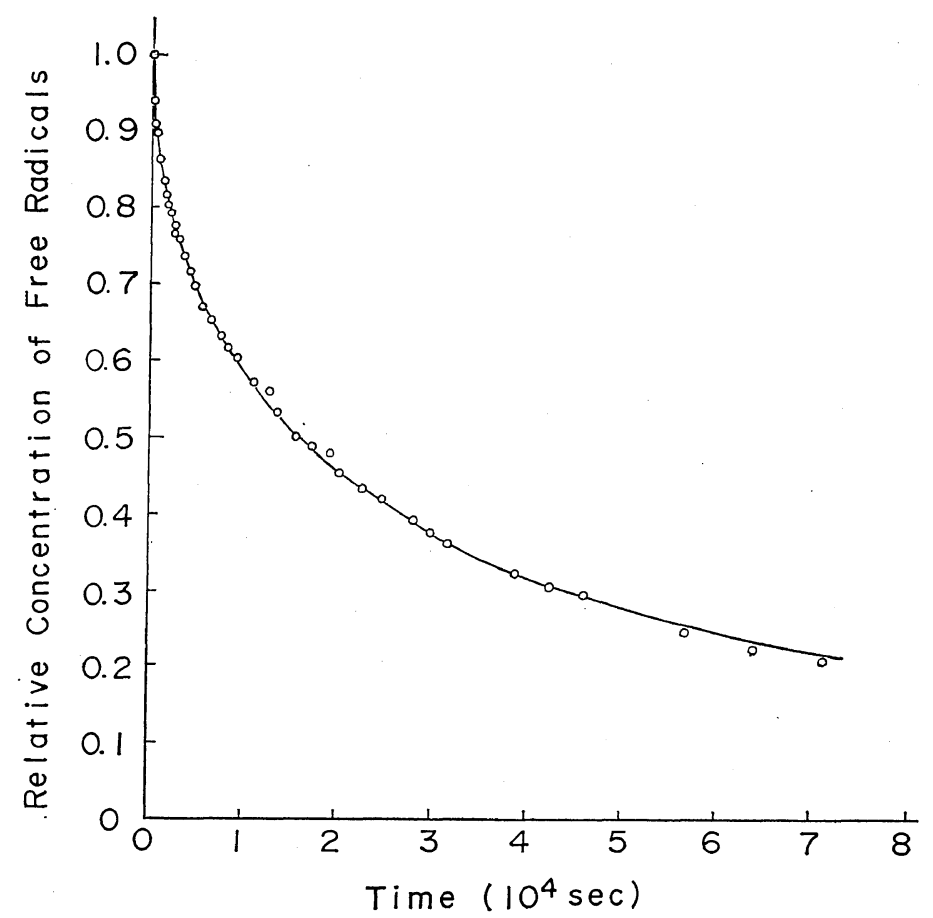

Figure 1. Variation of concentration of alkyl free-radical in polyethylene with time for the reaction at $27^{\circ} \mathrm{C}$ : open circle, estimated value from the observed ESR intensity; solid curve, calculated curve from eq $3 b$. 


\section{S. Shimada and H. Kashiwabara}

value of $a$. This value was found to be almost zero for the cases of higher-temperature measurements including the case of $27^{\circ} \mathrm{C}$, but it was found to be 0.62 for $-51^{\circ} \mathrm{C}$ and 0.44 for $0^{\circ} \mathrm{C}$. By using the value of $a$ estimated in this way, we can obtain the approximate values of $A$ and $B$ in eq 3 from the plot of $(1-a) /(x-a) v s . \sqrt{t}$ for shorter $t$ and that of $(1-a) /(x-a) v s$. $t$ for longer $t$. For the measurement at $27^{\circ} \mathrm{C}$, however, eq 3 can actually be applied for the estimations of $A$ and $B$ because of the negligible value of $a$. Starting from these values of $A$ and $B$, we can use an iteration process for the determination of the best values in order to obtain good agreement between the observed data and calculated curve of eq 3. Data for the measurements at $27^{\circ} \mathrm{C}$ were thus arranged and the values of $A$ and $B$ were found to be $3.7 \times 10^{-3}$ and $3.5 \times 10^{-5}$, respectively. The result is shown in Figure 1, in which open circles represent the plots of experimental data and solid curve indicates the calculated curve of eq 3 .

It can be said that analysis of the data based on the diffusion-controlled reaction theory makes an improvement concerning the interpretation of the situation of the decay reaction. $A$ and $B$ are, of course, temperature-dependent quan- tities, and, therefore, we can obtain the activation energy from the estimation of the diffusion constant at various temperatures. These estimations and more detailed discussions including the data at various temperatures will appear in a forthcoming publication. In this paper, we wanted to present an example of the good agreement between experimental plots and calculated curves as shown in Figure 1. An example of the analysis of the radical decay in polyethylene based on the diffusion-controlled process theory was also presented recently, ${ }^{4}$ but it was concerned with the case of the reaction of the radicals with oxygen molecules and the present analysis is concerned with the much simpler situation; i.e., only the decay of the radicals was considered.

\section{REFERENCES}

1. For example, S. Nara, S. Shimada, H. Kashiwabara, and J. Sohma, J. Polym. Sci. Part A-2, 6, 1435 (1968).

2. For example, R. Cracco, A. J. Arvia, and M. Dole, J. Chem. Phys., 37, 2499 (1962).

3. T. R. Waite, Phys. Rev., 107, 463 (1957)

4. T. Seguchi and N. Tamura, J. Phys. Chem., 77, 40 (1973). 